\title{
Guillain-Barr'E Syndrome with Bilateral Extensor Plantar Reflexes
}

\author{
Ahmad SF ${ }^{* 1,2}$, Al-Bader SY ${ }^{3}$ and Al-Hashel JY ${ }^{1,4}$ \\ ${ }^{1}$ Department of Neurology, Ibn Sina Hospital, Kuwait \\ ${ }^{2}$ Department of Neurology and Psychiatry, Al Minia University, Faculty of Medicine, Egypt \\ ${ }^{3}$ Department of Medicine, Mubarak Al-Kabeer Hospital, Kuwait \\ ${ }^{4}$ Department of Medicine, Kuwait University, Faculty of Medicine
}

*Corresponding author: Ahmad SF, Department of Neurology and Psychiatry, Al Minia University, Egypt, Fax No. 00965- 24849226, Tele: 00965- 24840837, 00965-60300562, E-mail: samerelshayb@hotmail.com

Citation: Ahmad SF, Al-Bader SY, Hashel JY (2014) Guillain-Barr'E Syndrome with Bilateral Extensor Plantar Reflexes. J Neurol Neurol Disord 1(1): 105 . doi: 10.15744/2454-4981.1.105

Received Date: November 14, 2014 Accepted Date: December 23, 2014 Published Date: December 30, 2014

\begin{abstract}
Background: Guillain-Barr'e syndrome (GBS) usually presents with lower motor neuron (LMN) symmetrical weakness, areflexia, and hypotonia. GBS has rarely been reported with up going plantars' response. We report a case of GBS with up going plantars during the course of the disease.

Case presentation: A 52-year-old woman developed acute paralysis with areflexia following a diarrheal illness. There was no fever at the time of the onset. She developed numbness and asymmetrical weakness of both lower limbs which progressed to involve the upper limbs bilaterally. There were no cranial nerves, respiratory or sphinecteric involvements. Few days later, the plantars' became upgoing bilaterally, with absent of other upper motor neuron signs. Cerebrospinal fluid (CSF) analysis showed albuminocytological dissociation. Early Nerve conduction study (NCS) showed absent H-reflex dispersed F-waves with prolonged maximum F-latencies and M-amplitude showed no clear block. MRI brain and spine were unremarkable. She was treated with intravenous immunoglobulin (IVIG). Repeated NCS two weeks after the onset, definitely confirmed conduction block. She was able to walk with support three weeks after the onset.

Conclusion: GBS should be considered as a differential diagnosis in a patient with acute quadriparesis, even if there is asymmetrical muscle weakness and extensor plantars" response.
\end{abstract}

Keywords: Guillain-Barr'e syndrome (GBS); Upgoing plantars'

\section{Introduction}

Guillain-Barré syndrome (GBS) is an acute autoimmune polyradiculoneuropathy. Clinical features include progressive, symmetrical ascending muscle weakness usually of more than two limbs, and areflexia with or without sensory, autonomic or brainstem involvements. Weakness is prominent in the lower limbs muscles compared to the upper limbs; there is absence of fever at the onset of neural symptoms [1]. Although, the diagnosis of GBS is based on clinical criteria, the presence of suggestive findings in the nerve conduction studies (NCS) or albuminocytological dissociation in the cerebrospinal fluid (CSF) analysis help to confirm the diagnosis [2].The involvement of the central nervous system (CNS) in the GBS is rare [3]. We report a case of GBS with asymmetrical weakness and up going plantars' during the course of the disease.

\section{Case presentation}

A 52-year-old female, known case of diabetes, hypertension, and hypothyroidism, was admitted with acute weakness of both lower limbs. She had history of diarrhea 4 days prior to presentation, which lasted 2 days and improved with symptomatic treatment. Then she developed numbness and weakness of both lower limbs that involved the left side more than the right. The weakness started distally and ascended up proximally. The weakness started to involve the upper limbs 4 days later. There were no cranial nerves, respiratory muscles involvement, nor sphincteric symptoms. There was no fever at the time of onset and no history of recent vaccination. On examination, there was weakness of both lower limbs with hypotonia. The weakness of both lower limbs was asymmetrical with proximal muscle power of 2/5 (Medical Research Council grading) in the left side and 4/5 in the right side, while the power of the distal muscles was $1 / 5$ in the left side and 3/5 in the right side. Examination of the upper limbs showed weakness of distal muscles of 4/5. Deep tendon reflexes were absent all over, and the plantars' were flexors bilaterally. Decrease in fine touch and temperature sensations were seen in L4, L5, S1 distribution of the left side with loss of joint position and vibration sense bilaterally. There was no cranial nerves involvement. The investigations showed normal total and differential leukocyte counts, erythrocyte sedimentation rate $(4 \mathrm{~mm} / \mathrm{h})$, creatine kinase level, and electrolytes ( $\mathrm{Na}, \mathrm{Ca}, \mathrm{K}, \mathrm{Mg}$, phosphate). Vasculitis and paraneoplastic work up were all negative. CSF examination showed albuminocytologic dissociation. CSF sugar was $6.3 \mathrm{mmol} / \mathrm{L}$ (plasma glucose $11 \mathrm{mmol} / \mathrm{L}$ ), protein was $1517 \mathrm{mg} / \mathrm{L}$, and there are only 2 cells (100\% lymphocytes) were detected. All causes of 
Infectious radiculoneuropathies were ruled out by analysis of serologies and PCRs for infectious agents. Magnetic resonance imaging (MRI) of lumbosacral spine showed L4/L5 level diffuse posterior disc bulge, and L5/S1 paracentral disc protrusion. MRI of the brain was normal. Nerve conduction study (NCS) in the first day of admission (which is the second day of onset) showed evident of L4, L5 and S1 radiculopathy on the left side and absent of H-reflex with mild axonal peripheral neuropathy (Table 1). Based on the clinical suspicion of GBS and CSF analysis, the patient was treated with IVIG (400 mg/kg/day) for $5 \mathrm{days}$. Regular physiotherapy was started. Three days later, the plantars' were both extensors without other signs of upper motor neurons. Repeated MRI spine was done to rule out transverse myelitis versus a cord lesion, and it showed no new changes.

\begin{tabular}{|c|c|c|c|}
\hline F wave latency (ms) & On the second day of the onset & On the sixth day of the onset & Two weeks after the onset \\
\hline Lt peroneal nerve & 42.1 & 49.0 & 55.2 \\
\hline Rt posterior tibial nerve & 48.8 & 53.8 & 42.0 \\
\hline Lt median nerve & 22.2 & 31.2 & 46.0 \\
\hline Rt ulnar nerve & 23.9 & 33.2 & 45.8 \\
\hline Distal latency (ms) & On the second day of the onset & On the sixth day of the onset & Two weeks after the onset \\
\hline Lt peroneal nerve & 4.6 & 4.4 & 7.2 \\
\hline Rt posterior tibial nerve & 4.5 & 4.6 & 4.3 \\
\hline Lt median nerve & 3.7 & 3.9 & 3.7 \\
\hline Rt ulnar nerve & 2.9 & 3.2 & 3.6 \\
\hline M wave amplitude ( $m v)$ & On the second day of the onset & On the sixth day of the onset & Two weeks after the onset \\
\hline Lt peroneal nerve & 3.2 & 1.5 & 1.2 \\
\hline Rt posterior tibial nerve & 7.5 & 5.8 & 6.6 \\
\hline Lt median nerve & 9.2 & 6.9 & 5.4 \\
\hline Rt ulnar nerve & 8.2 & 6.0 & 5.4 \\
\hline Motor conduction velocity $(\mathrm{m} / \mathrm{s})$ & On the second day of the onset & On the sixth day of the onset & Two weeks after the onset \\
\hline Lt peroneal nerve & 45.0 & 42.0 & 25.0 \\
\hline Rt posterior tibial nerve & 51.0 & 50.0 & 42.0 \\
\hline Lt median nerve & 56.0 & 42.0 & 32.0 \\
\hline Rt ulnar nerve & 56.0 & 42.0 & 30.0 \\
\hline Sensory conduction velocity $(\mathrm{m} / \mathrm{s})$ & On the second day of the onset & On the sixth day of the onset & Two weeks after the onset \\
\hline Lt median nerve & 48.0 & 48.0 & 43.0 \\
\hline Rt ulnar nerve & 54.0 & 50.0 & 42.0 \\
\hline Lt sural nerve & 54.0 & 43.0 & 46.0 \\
\hline
\end{tabular}

${ }^{*}$ Lt: Left; Rt: Right; ms: millisecond, mv: millivolt, m/s: meter/second.

Table 1: Electrodiagnostic tests of the GBS case

Repeated NCS (six days from the onset), showed absent of H-reflex and dispersed of F-waves with prolonged maximum F-latencies. M-amplitude is lower than normal but no clear block. Bilateral sural sensory conduction velocity was normal. Third NCS (two weeks from the onset) showed a clear conduction block (reduction of amplitude less than 50\%) with slowing absent or delayed F- latencies in the lower limbs. Drop in amplitude, slowing and late response changes were seen in the upper limbs. Sensory responses were decreased compared to previous study (Table 1). Somatosensory evoked potentials were normal. Five days later, the plantars became flexors again. The patient improved and was able to walk with minimal support three weeks after the onset.

\section{Discussion}

This is a case of GBS with an uncommon clinical presentation, characterized by flaccid quadriplegia with asymmetrical weakness and bilateral up going plantars' response. A careful history of acute weakness that was preceded by a history of diarrhea, with areflexia and albuminocytological dissociation in CSF examination raised the suspicion to consider the diagnosis of GBS. Despite an atypical pattern of clinical signs and symptoms, IVIG therapy was started on the day of admission which leaded to the functional recovery of our patient.

Our case is similar to the case of Cattano and his colleagues [4] who presented a 66-year-old non-insulin-dependent diabetic man with global muscle weakness more in the lower limbs, with upper- and lower-limbs areflexia. There were no sensory or cranial nerve deficits. Unexpectedly, his clinical examination showed a right positive Babinski sign. Their diagnosis of GBS was confirmed with neurophysiological study. Our case is also similar to the case of Kiriyama and his colleagues [5] who presented a 30-year-old male patient who was diagnosed with GBS based on the presentation of acute flaccid weakness and a recent history of diarrhea. The weakness was asymmetrical and they assumed that there is a central involvement. The diagnosis was later confirmed with abnormal motor evoked potential (MEP) bilaterally and with positive anti-C. jejuni and anti-ganglioside antibodies. 
The most common differential diagnosis with acute progressive weakness and up going plantars' response is myelopathy. It was excluded in our case with the absence of sphincteric symptoms. A spinal MRI finding in our case was disc lesion at the level of L4/L5 and L5/S1, which are chronic degenerative changes and did not explain these clinical findings. Our patient developed acute weakness which involved distal and proximal muscles of both lower limbs and distal muscles of the upper limbs. The proximal muscles of the lower limbs (flexors of the hip) are supplied by L1 root, and the distal muscles of the upper limbs are supplied by C8, T1 roots which are not involved in these degenerative lesions.

GBS is characterized by symmetrical lower motor neuron weakness [1]. In our case, the weakness was asymmetrical and there was bilateral up going plantars which may suggest central involvement of nervous system (CNS). The peripheral involvement in GBS may have partly masked the central involvement typically associated with hyperreflexia. Previous pathological and electrophysiological studies of GBS have demonstrated the involvement of the CNS, where the clinical relevance remains uncertain [6, 7]. Other autopsy studies had also provided evidence of CNS involvement in patients with GBS which includes: central chromatolysis of motor neurons, mononuclear cell filtration, and microglial activation in the lateral column of the spinal cord [6]. There was another study suggested CNS involvement in GBS. They concluded that prolongation in central conduction time in both Brainstem auditory evoked potentials (BAEPs) and Visual evoked potentials (VEPs) suggested the subclinical auditory and optical pathway involvement in GBS [8].

Electro-physiological hallmarks of early demyelination include: prolonged distal motor latencies, prolonged/absent F wave latencies (mainly in the lower limbs), slow motor conduction velocities/conduction block (CB), absent F wave, and abnormal sensory nerve action potential in the upper extremities compared to the sural nerve. F wave is the most sensitive diagnostic test for early GBS [1]. In our case, prolonged $\mathrm{F}$ wave latency was detected early in the course of the illness which supported our clinical diagnosis of GBS. CB is more frequently observed in the early stage of GBS, when it may be the only sign of demyelination and the main reason of acute paralysis in GBS [9]. CB indirectly determined as absent $\mathrm{H}$ reflex which was recorded early in our case. It was the most commonly observed abnormality in the early stage of GBS in previous studies $[9,10]$.

The electrophysiological findings may vary in the same patient during the course of the disease, and the characteristical abnormalities may not evolve for several days or weeks [11] as it was seen in our case. First electrophysiological study (on second day of onset) showed mildly prolonged $\mathrm{F}$ wave latencies in the lower limbs. The second one (on sixth day of onset) showed more prolongation of $\mathrm{F}$ wave latencies with the absence of $\mathrm{H}$ reflexes (which suggested GBS). The third study (after 2 weeks from the onset) confirmed the diagnosis of GBS.

\section{Conclusion}

It should be noted that, patients with GBS may present with signs and symptom associated with CNS involvement. Although the presence of up going plantars' response and asymmetrical weakness in GBS is rare, the treating physicians and neurologists should have a high grade of suspicion toward the diagnosis of GBS especially if the patient presents with acute areflexic weakness. Tests like NCS and CSF analysis can confirm the clinical findings. Early diagnosis and treatment of GBS may prevent permanent disability and mortality.

\section{Acknowledgment}

We are grateful to the neurophysiology department in Ibn Sina Hospital, Kuwait and Dr. Rossen T. Rooussef, the neurophysiologist.

\section{References}

1. Geetanjali S, Sushma S, Sudhir S (2013) Early Electrodiagnostic Findings of Guillain Barre Syndrome. J Neurol Neurophysiol 4: 142.

2. Van Doorn PA, Ruts L, Jacobs BC (2008) Clinical features, pathogenesis, and treatment of Guillain-Barré syndrome. Lancet Neurol 7: 939-50.

3. Hughes RA, Cornblath DR (2005) Guillain-Barre syndrome. Lancet 366: 1653-66.

4. Cattano D, O'connor B, Shakir R, Palazzo M (2008) Acute Inflammatory Demyelinating Polyneuropathy and a Unilateral Babinski/Plantar Reflex. Anesthesiol Res Pract 1155: 134958.

5. Kiriyama T, Hirano M, Kusunoki S, Uneo S, Morita D et al. (2009) Asymmetrical Weakness Associated with Central Nervous System Involvement in a Patient with Guillain-Barrè Syndrome. Clinical Medicine: Case Repo 2: 51-4.

6. Maier H, Schmidbauer M, Pfausler B, Budka K, Schmidbauer M (1997) Central nervous system pathology in patients with the Guillain-Barre syndrome. Brain 120: 451-64.

7. Oshima Y, Mitsui T, Yoshino H, Asano A, Endo I, et al. (2002) Central motor conduction in patients with anti-ganglioside antibody associated neuropathy syndromes and hyperreflexia. J Neurol Neurosurg Psychiatry 73: 568-73.

8. Ghildiyal A, Shraddha S, Iqbal B, Verma P, Tiwari S, et al. (2012) Central Demyelination in Guillain-Barre syndrome. Current Neurobiology 3: 117-22.

9. Atanasova D, Ishpekova B, Muradyn N, Novachkova S, Daskalov M (2004) Conduction block - the diagnostic value in the early stage of Guillain-Barré syndrome. Electromyogr Clin Neurophysiol 44: 361-4.

10. BarabaR, Sruk A, Šragalj L, Bielin I, Butković-Soldo S (2011) Elec trophysiologicall Findings in Early Guillain-Barré Syndrome. Acta Clin Croat 50: $201-207$. 11. Albers JW, Donofrio PD, Mcgonagle TK (1985) Sequential electrodiagnostic abnormalities in acute inflammatory demyelinating polyradiculoneuropathy. Muscle Nerve 8: 528-39. 


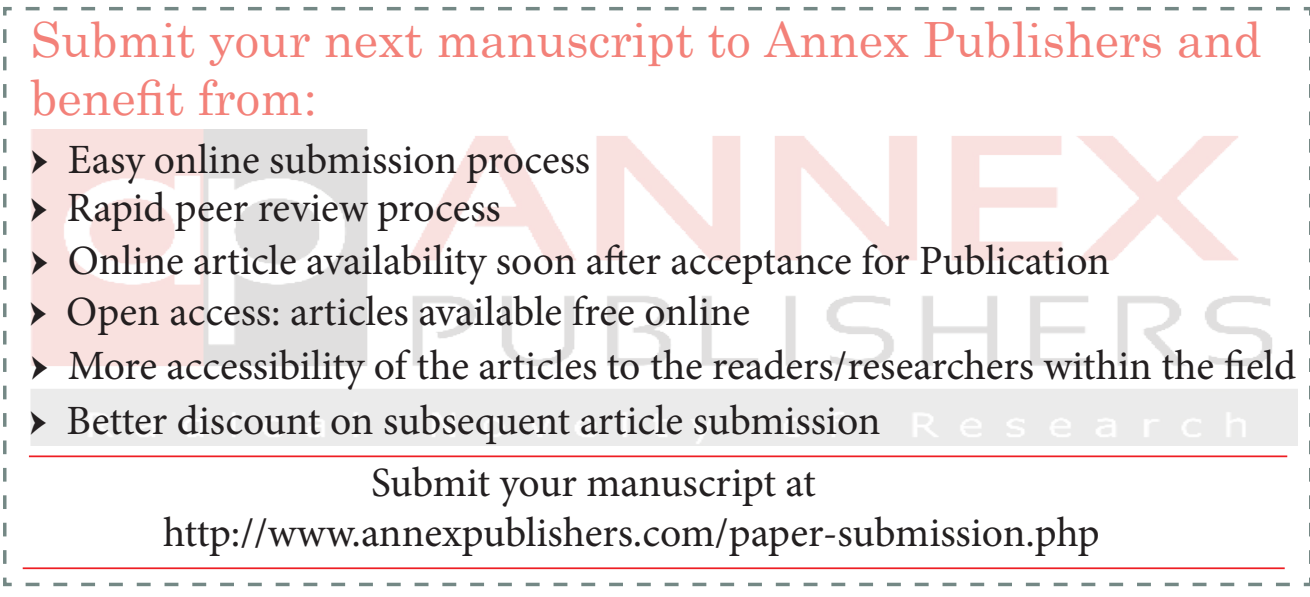

